\title{
Relevanz sprachlicher \\ Kompetenzen im \\ Anästhesistenberuf in Deutschland
}

\section{Einleitung}

Welche Zukunft haben Sprachen wie Deutsch in einer zunehmend anglophonen Wissenschaftswelt? Welche Relevanz hat Mehrsprachigkeit noch in der akademischen Bildung? In diesem Beitrag soll der Blick über das Studium hinaus auf die Berufspraxis von Akademikern/innen gelenkt werden. Am Beispiel eines authentischen anästhesiologischen Aufklärungsgesprächs zwischen einer ausländischen Anästhesistin und ihrem Patienten wird die hohe Relevanz von differenzierten Deutschkenntnissen in diesem akademischen Beruf aufgezeigt. Grundlage für diesen Artikel bildet Daten, die im Rahmen der Studie Sprachliche Herausforderungen ausländischer Anästhesisten/innen bei Aufklärungsgesprächen ${ }^{1}$ erhoben wurden.

$1 \quad \mathrm{Zu}$ der Studie liegt bereits eine gleichnamige Monografie vor (Borowski 2018), in der die Ergebnisse dreier Einzelfallanalysen (Kapitel 5, 6 und 7) und die Ergebnisse der Analyse einer Sammlung (Kapitel 8) präsentiert werden. Bei den Einzelfällen handelt es sich um drei anästhesiologische Aufklärungsgespräche (A101/P102, A102/P106 und A103/ P110), die in ihrer Gesamtheit betrachtet werden. Bei der Sammlung geht es um alle Vorkommen des Phänomens >Verstehenssicherung ‘ in den zwölf dokumentierten Gesprächen. Das Gespräch zwischen A101 und P104 wird in dem
In diesem Beitrag wird die Bedeutung von Mehrsprachigkeit in der akademischen Bildung am Beispiel eines videografierten Aufklärungsgesprächs zwischen einer ausländischen Anästhesistin und ihrem Patienten aufgezeigt. Aus der Transkript-Analyse geht hervor, dass die eingeschränkten Sprachkompetenzen der Ärztin ihre Fähigkeit stark beeinträchtigen, das Gespräch angemessen zu führen. Dieser Blick in den Berufskontext einer der derzeit annähernd 55.000 ausländischen Ärzten/innen in Deutschland zeigt die (in diesem Fall medizinische und rechtliche) Relevanz der Beherrschung der Nationalsprache in akademischen Berufen - speziell, wenn ExpertenLaien-Kommunikation zum Berufsalltag gehört. 
Die Diskussion um den Fachkräftemangel in Deutschland hat in dem kürzlich von der Bundesregierung vorgelegten Entwurf zum Fachkräfteeinwanderungsgesetz ${ }^{2}$ einen weiteren Höhepunkt erreicht. In dieser Diskussion sind die Gesundheitsberufe kontinuierlich ein zentrales Thema. »Wir zehren seit Jahren von der Substanz. Die Zahl der Ärztinnen und Ärzte wächst zu langsam, um die enormen Herausforderungen zu bewältigen, vor denen unser Gesundheitssystem steht. « ${ }^{3}$ Mit diesen Worten beklagt Montgomery (Präsident der Bundesärztekammer) bei der Vorstellung der Ärztestatistik 2018 den Ärztemangel in Deutschland. Im Kampf gegen den Ärztemangel spielt die Anwerbung von ausländischen Ärzten/innen eine wichtige Rolle. Die Ärztestatistik zeigt, dass die Zahl der zugewanderten Ärzte/innen auch im Jahr 2018 weiter gestiegen ist (im Vergleich zum Vorjahr um ca. 3.500). Die annähernd 55.000 ausländischen Ärzte/innen kommen vorrangig aus Rumänien (4.312), Syrien (3.908), Griechenland (2.777) und Österreich (2.309). Immer wieder werden die sprachlichen Kompetenzen dieser Ärzte/innen in den Medien diskutiert. In der linguistischen Forschung sind sprachliche Kompetenzen von Ärzten/innen in der Berufspraxis allerdings bisher kaum betrachtet worden.

Die Arzt-Patienten-Interaktion ist seit den 1970er-Jahren ein etabliertes Forschungsfeld in der Linguistik und hat zahlreiche Studien mit unterschiedlichen theoretischen und methodischen Ansätzen hervorgebracht. ${ }^{4}$ Auch zur Mehrsprachigkeit in der Arzt-Patienten-Interaktion liegen bereits zahlreiche Studien vor. ${ }^{5}$ Einen aktuellen Forschungsüberblick zur ArztPatienten-Interaktion im deutschsprachigen Raum und zur Mehrsprachigkeit in der Arzt-Patienten-Interaktion gibt Borowski. ${ }^{6}$ Zusammenfassend werden folgende zentrale Forschungsergebnisse festgehalten:

Sprachliche Barrieren können zu einer Vereinfachung oder Reduktion der Gesprächsinhalte führen. Diese Barrieren sowie unterschiedliche Vorstellungen in Bezug auf Rollen, Skripte und Abläufe verkomplizieren die Interaktion. Sprachliche und kulturelle Differenzen haben zum Teil einen medizinisch oder rechtlich relevanten Einfluss auf die Gespräche. ${ }^{7}$

Diese Ergebnisse beziehen sich allerdings ausschließlich auf Studien mit Patienten/innen, die Deutsch bzw. Englisch als Zweitsprache sprechen.

vorliegenden Beitrag erstmalig in seiner Gesamtheit betrachtet. In der Monografie fließen lediglich Auszüge dieses Gesprächs in der Analyse der Sammlung zur »Verstehenssicherung« mit ein.

3 Ergebnisse der Ärztestatistik zum 31. Dezember 2018.

$4 \quad$ Menz et al.: Arzt-Patienten-Interaktion im deutschsprachigen Raum, S. 130.

5 Bührig/Meyer: Ärztliche Gespräche mit MigrantInnen, S. 302.

6 Borowski: Sprachliche Herausforderungen, S. 40-51.

7 Ebd., S. 47. 
Interaktionen mit Ärzten/innen, die Deutsch als Zweitsprache sprechen, haben bisher in der linguistischen Forschung kaum Beachtung gefunden. Gleichwohl ist es nicht zuletzt ein Gebot der Patientensicherheit, angemessene Anforderungen an Deutschkenntnisse von Ärzten/innen zu stellen. Die Studie von Borowski ist die erste, die authentische Arzt-PatientenInteraktion mit Ärzten/innen, die Deutsch als Zweitsprache sprechen, in den Blick nimmt. Im Folgenden werden zunächst einige relevante Hintergrundinformationen gegeben (2). Im Anschluss wird ein einzelnes Gespräch (Ärztin A101 und Patient P104) im Detail betrachtet (3).

\section{Hintergrundinformationen}

\section{Zugrundeliegende Studie}

Das Erkenntnisinteresse der Studie ${ }^{8}$ hat sich in der Unterrichtspraxis Deutschkurse für Ärzte/innen - entwickelt. Als zentrales Problem in diesem Vermittlungskontext wurde die noch immer mangelnde Kenntnis der tatsächlichen sprachlichen Herausforderungen ausländischer Ärzte/innen im Krankenhausalltag identifiziert. ${ }^{9}$ Aufgrund der fehlenden Forschung weisen die vorliegenden Materialien und Konzepte erhebliche Mängel auf.

Ziel der Studie war es, den Einfluss mangelnder Deutschkompetenzen von Anästhesisten/innen mit Deutsch als Zweitsprache auf anästhesiologische Aufklärungsgespräche zu erfassen. Dabei wurde das Forschungsfeld der Gesprächsanalyse mit dem der Zweitspracherwerbsforschung verbunden. Für die Studie wurden im laufenden Klinikalltag 12 anästhesiologische Aufklärungsgespräche videografiert. Die teilnehmenden Ärzte/innen hatten ihre Studienabschlüsse in Ägypten, Rumänien und Italien erlangt und zum Zeitpunkt der Datenerhebung bereits 3 bis 5 Jahre lang in Deutschland gearbeitet.

Um zu erfassen, welche sprachlichen Fehler in den Äußerungen der Anästhesisten/innen auftauchen, wurde eine Fehleranalyse ${ }^{10}$ durchgeführt. In einem weiteren Schritt wurde die Konversationsanalyse ${ }^{11}$ (Einzelfallanalyse und Kollektion) eingesetzt, um zu erfassen, wie die Gespräche verlaufen und welche interaktiven Auffälligkeiten und Störungen auftreten. Diese

8 Borowski: Sprachliche Herausforderungen.

9 Ebd., S. 52.

10 Baur/Chlosta/Schäfer: Fehleranalyse.

11 Gülich/Mondada: Konversationsanalyse, S. 18. 
beiden Analyseverfahren wurden (anders als bei Barkhuizen/Ellis) ${ }^{12}$ getrennt voneinander eingesetzt und die Ergebnisse im Anschluss miteinander in Beziehung gesetzt. Um die verschiedenen Gespräche miteinander und mit bereits vorliegenden Studienergebnissen vergleichen zu können, wurde zusätzlich eine Systematisierung der Gesprächsverläufe mithilfe von Nowaks Systematik ${ }^{13}$ vorgenommen. Auf diese Weise konnten Zusammenhänge zwischen sprachlichen Fehlern und Störungen in den Gesprächen nachvollzogen werden. Ergänzend zu den Primärdaten wurden verschiedene Sekundärdaten (Feldnotizen, Aufklärungs- und Anamnesebögen, Auszüge aus den Patientenakten, Anästhesieprotokolle) erhoben, die zur Erweiterung des Verständnisses dieses hochkomplexen Gesprächstyps dienen. Dabei wurde auch die Perspektive der Patienten/innen und Anästhesisten/innen durch Interviews einbezogen. Vor diesem Hintergrund kann die Studie als gesprächsanalytische Studie mit ethnomethodologischer Rahmung bezeichnet werden.

Die Studie kommt zu dem Ergebnis, dass die eingeschränkte Sprachkompetenz von Anästhesisten/innen ihre Fähigkeit, die Aufklärungsgespräche angemessen zu führen, stark beeinträchtigt. Die Analyse lässt sichtbar werden, dass unterschiedliche sprachlich-kommunikative Fehlleistungen der Ärzte/innen beachtliche Störungen im Interaktionsgeschehen hervorrufen. So werden Gründe für die Beeinträchtigung und vielfach sogar für das Scheitern der vorgesehenen Gesprächsabwicklungen aufgezeigt. In der Studie wird ferner gezeigt, dass in den analysierten Gesprächen unzureichende Verstehenssicherung keine Ausnahme ist.

\section{Das anästhesiologische Aufklärungsgespräch}

Anästhesiologische Aufklärungsgespräche sind rechtlich vorgeschriebene Arzt-Patienten-Gespräche zur Vorbereitung operativer Eingriffe. ${ }^{14}$ Dabei handelt es sich um institutionalisierte mündliche Kommunikation, die sich zu großen Teilen an schriftlichen Unterlagen orientiert (Aufklärungs- und Anamnesebögen sowie Patientenakten). Aufgrund der rechtlichen und medizinischen Anforderungen haben anästhesiologische Aufklärungsgespräche ein hohes Maß an Standardisierung und müssen individualisiert und dokumentiert (Anästhesieprotokoll) werden. Im Rahmen der Studie 
konnten - auf der Grundlage von Nowaks Systematik ${ }^{15}$ - sechs Standardkomponenten dieses Gesprächstyps identifiziert werden: Eröffnung, Anamnese, ggf. körperliche Untersuchung, Aufklärung, Einwilligung und Abschluss. In der Abbildung 1 wird der übliche Ablauf der Gespräche veranschaulicht und Datenbeispiele für die verschiedenen Komponenten gegeben. Die Abbildung 1 zeigt auch, dass die Gespräche in der Regel mehrfach durch Telefonate (krankenhausinterne Anrufe auf die Arbeitshandys der Anästhesisten/innen) gestört werden.

\section{Transkriptionszeichen}

Die Transkription orientiert sich an dem Gesprächsanalytischen

Transkriptionssystem GAT2. ${ }^{16}$ Folgende Transkriptionszeichen wurden verwendet:

\begin{tabular}{|l|l|}
\hline$()$. & Minipause \\
$(-)$ & geschätzte Pause unter einer Sekunde \\
$(--)$ & geschätzte Pause über einer Sekunde \\
$(0.5)$ & gemessene Pause \\
und_h hm_hm & Verschleifung \\
$=$ & unmittelbarer Anschluss eines neuen Segments (latching) \\
'hm 'hm & mit Glottalverschlüssen (meist verneinend) \\
$:$ & Dehnung \\
ne: & Verneinung \\
ne $\uparrow$ & tag-question \\
äh öh & gefüllte Pausen \\
haha hehe hihi & silbisches Lachen (der Deskription vorgezogen) \\
() & unverständliche Passage \\
$(\mathrm{XXX})$ & unverständliche Passage mit Silbenanzahl \\
$(\mathrm{XXX} . .)$. & unverständliche Passage mit mehreren Silben, Anzahl unklar \\
$\mathrm{XXX}$ & Namen (abgesehen von A und P) mit Silbenanzahl \\
$($ wichtig) & vermuteter Wortlaut \\
$($ wichtig/nichtig) & mögliche Alternativen \\
akZENT & verhältnismäßig auffällige Akzentuierung \\
$\uparrow$ & Intonation steigend (der Verwendung von Fragezeichen vorgezogen) \\
$\downarrow$ & Intonation fallend \\
$\rightarrow \rightarrow$ & Intonation gleichbleibend (meist turn noch nicht beendet) \\
$\mathrm{h}^{\circ}$ & Ausatmen \\
${ }^{\circ} \mathrm{h}$ & Einatmen \\
$<<$ p $>>$ & verhältnismäßig leise \\
$<<\mathrm{f}>>$ & verhältnismäßig laut \\
\hline
\end{tabular}

15 Nowak: Eine Systematik der Arzt-Patient-Interaktion.

16 Selting et al.: Gesprächsanalytisches Transkriptionssystem 2 (GAT 2). 


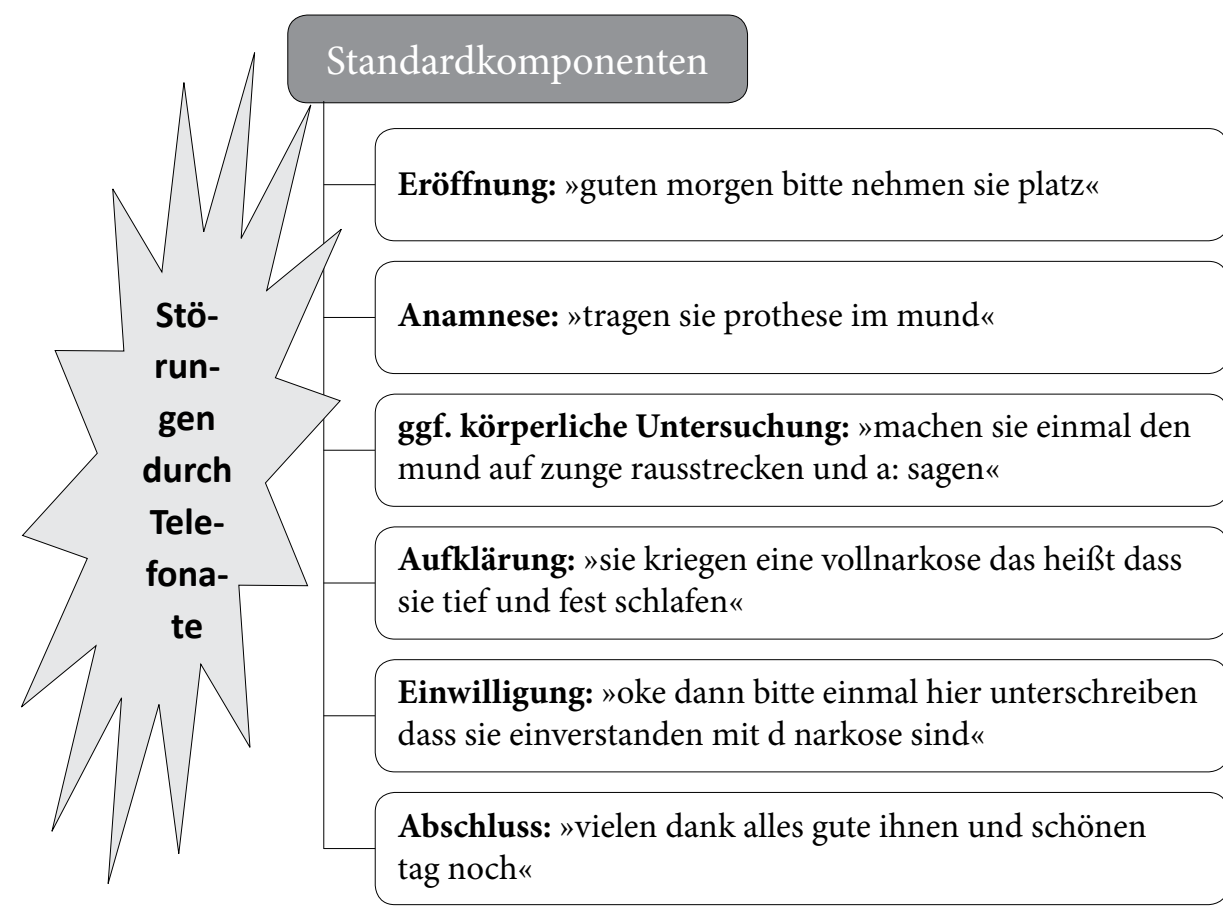

Abbildung 1: Standardkomponenten anästhesiologischer Aufklärungsgespräche

Folgende zentrale Handlungsziele sind für die anästhesiologische Aufklärung festgelegt:

Anamnese: Die Ärzte/innen müssen im Gespräch die Informationen über den Gesundheitszustand der Patienten/innen ermitteln, die für die Anästhesie relevant sind. ${ }^{17}$ Dazu kann auch eine körperliche Untersuchung nötig sein.

Entscheidung: Im Rahmen des Gespräches müssen die Anästhesisten/innen eine Entscheidung über das anzuwendende Anästhesieverfahren treffen und ggf. eine Prämedikation (Medikation zur Angstlösung und Sedierung vor der eigentlichen Anästhesie) verordnen. ${ }^{18}$ Kommen mehrere Verfahren in Frage, soll im Sinne des >shared decision making ein Austausch an medizinisch relevanter Information durch die Ärzte/innen und individueller Sichtweisen durch die Patienten/innen stattfinden und auf dieser Grundlage eine konsensuelle Entscheidung getroffen werden. ${ }^{19}$

Aufklärung/Information und Einwilligung: Letztlich ist das Ziel dieser Gespräche das Einholen der rechtlich wirksamen Zustimmung der

18 Ebd., S. 3.

19 Nowak: Eine Systematik der Arzt-Patient-Interaktion, S. 325f. 
Patienten/innen zu einem bestimmten Anästhesieverfahren. Die Anästhesisten/innen sind hierzu verpflichtet, ihre Patienten/innen angemessen über das geplante und alternative Verfahren aufzuklären und gegenseitiges Verständnis zu sichern. ${ }^{20}$ Löning hebt in diesem Zusammenhang besonders hervor, dass die Information zu einer »verantwortlichen Entscheidung beim Patienten « und zur »Herstellung eines rechtserheblichen Konsens zwischen Arzt und Patient « führen muss. ${ }^{21}$ Im Englischen wird hier treffend die Bezeichnung informed consent verwendet. $^{22}$

Verringerung von Angst/Beruhigung: Eng mit der Aufklärung/ Information verbunden ist die Intention, die Patienten/innen zu beruhigen, bzw. ihre Angst zu verringern. So formuliert Striebel als zentrales Ziel der Gespräche: »Aufklärung des Patienten sowie Eingehen auf seine Fragen und Ängste bezüglich der Narkose «. ${ }^{23}$ Auch Larsen nennt die Verminderung von Angst und Aufregung als ein Ziel, das durch Aufklärungsgespräche erreicht werden soll. ${ }^{24}$

\section{Sprachliche Fehler im anästhesiologischen Aufklärungsgespräch}

Als sprachlicher Fehler wird in der Studie von Borowski (und dementsprechend auch in diesem Beitrag) alles verstanden, was auf der Ebene der Phonetik/Phonologie, Morphologie, Syntax, Lexik und/oder Pragmatik von der Norm des anästhesiologischen Aufklärungsgesprächs abweicht. ${ }^{25}$ Dabei werden Fehler nicht defizitär, sondern als Indikatoren eines Kompetenzniveaus verstanden. ${ }^{26}$ Die Norm definiert sich über den gegebenen Kontext: Es handelt sich zunächst um gesprochene Sprache. ${ }^{27}$ Darüber hinaus sind die Gespräche institutionalisiert, asymmetrisch und verfolgen die oben beschrieben Ziele. Vor diesem Hintergrund werden beispielsweise elliptische Fragen (z.B.:»Vorerkrankungen?«) nicht als grammatische Fehler, sondern als der Norm anästhesiologischer Aufklärungsgespräche entsprechend verstanden.

In diesem Beitrag spielt die >unangemessene Ausdrucksweise eine zentrale Rolle. Hierunter werden Fehler auf der Ebene der Pragmatik ver-

21 Löning: Gespräche in der Medizin, S. 1585.

22 Mann: Aufklärung in der Medizin, S. 8.

23 Striebel: Anästhesie - Intensivmedizin - Notfallmedizin, S. 3.

24 Larsen: Anästhesie, S. 307f.

25 Baur/Chlosta/Schäfer: Fehleranalyse.

26 Ebd.

27 Schwitalla: Gesprochenes Deutsch. 
standen, die Baur et al. treffend beschreiben als »falsche[s] Register, Stilbruch oder kulturell nicht angemessenes sprachliches Verhalten ${ }^{28}{ }^{28}$ Mit der Bezeichnung > unangemessen` wird in diesem Beitrag ausdrücklich keine Wertung vorgenommen, sondern eine Abweichung von der Norm des anästhesiologischen Aufklärungsgespräches festgestellt. In dem unten fokussierten Gespräch fällt eine bestimmte Form der >unangemessenen Ausdrucksweise besonders auf - die `beunruhigende Ausdrucksweise Es handelt sich dabei um Ausdrucksweisen, die von Patienten/innen als beunruhigend wahrgenommen werden können. Obwohl die Wahrnehmung einer Ausdrucksweise als beunruhigend oder beruhigend immer subjektiv ist, muss diesem Bereich der Pragmatik im anästhesiologischen Aufklärungsgespräch unbedingt Beachtung geschenkt werden. Oben wurde bereits dargestellt, dass die Verringerung von Angst ein zentrales Ziel dieser Gespräche ist. Dies kann nur gelingen, wenn die Inhalte durch eine möglichst beruhigende Ausdrucksweise übermittelt werden.

\section{Verstehenssicherung im anästhesiologischen Aufklärungsgespräch}

Um die oben genannten zentralen Handlungsziele zu erreichen, ist Verstehenssicherung nötig:

Die Absicherung des Wissens ist deshalb wesentlicher Bestandteil der Aufklärungsgespräche, da sich der Arzt schon allein aus rechtlichen Gründen darüber versichern muss, dass der Patient die Information verstanden hat. Der Arzt ist dazu angewiesen, dem Patienten Fragerechte einzuräumen. Nur wenn sich die Interaktionspartner gegenseitiges Verstehen versichern, ist die Basis für das Einverständnis geschaffen. ${ }^{29}$

Um Verstehen zu sichern, können Ärzte/innen und Patienten/innen verschiedene interaktive Verfahren einsetzen. ${ }^{30}$ In der folgenden Tabelle werden die Verfahren aufgelistet, die in dem unten fokussierten Aufklärungsgespräch zum Einsatz gekommen sind. Zur Veranschaulichung ist jeweils ein Beispiel aus den Daten gegeben. Die Beispiele sind hier zur Veranschaulichung aus dem Kontext gegriffen. Die Zuordnung der einzelnen Datenbeispiele zu einem Verfahren der Verstehenssicherung ist allerdings in einigen Fällen nur im Kontext möglich und daher an dieser Stelle nicht immer nachvollziehbar.

30 Klüber/Motsch/Spranz-Fogasy: Verständigungssicherung, S. 251-254; Nowak: Eine Systematik der Arzt-Patient-Interaktion, S. 273-278. 


\begin{tabular}{|l|l|}
\hline Fragen/Nachfragen & P104: »ist das denn sehr schmerzhaft diese spritze hier $\uparrow$ « \\
\hline Deklarativsatzfragen & P104: »also bin ich jetzt nicht hellwach die ganze zeit» \\
\hline Wiederholen & $\begin{array}{l}\text { P104: »ich denke bei der lokalen narkose ist das risiko noch } \\
\text { geringer « }\end{array}$ \\
\hline Rephrasieren & $\begin{array}{l}\text { P104: »also kriege ich erstmal denke ich irgendwo eine kleine } \\
\text { spritze und dann wird das taub und dann kriege ich die große } \\
\text { spritze hier« }\end{array}$ \\
\hline Zusammenfassen & $\begin{array}{l}\text { P104: »so wie ich das jetzt verstanden habe kriege ich en } \\
\text { beruhigungsmittel vor der ope: dann kriege ich hier einen tropf...» }\end{array}$ \\
\hline $\begin{array}{l}\text { Abschließende } \\
\text { Nachfragemöglichkeit }\end{array}$ & A101: »noch fragen daZU $\uparrow$ \\
\hline
\end{tabular}

Tabelle 1: Verfahren der Verstehenssicherung

Klüber et al. haben Verstehenssicherungen in anästhesiologischen Aufklärungsgesprächen mit Ärzten/innen, die Deutsch als Erstsprache sprechen, in den Blick genommen. ${ }^{31}$ Klüber fasst einige Ergebnisse dieser Studie wie folgt zusammen:

Im Durchschnitt stellen die Patienten in einem Gespräch 2,4 Fragen. 73,5 Prozent der Fragen sind jedoch eigeninitiativ. 80 Prozent der eigeninitiativen Fragen werden in der Aufklärungsphase gestellt. [...] Am häufigsten stellen die Patienten Deklarativsatzfragen (58,8 Prozent), die bereits ein relativ deutliches Wissen ausdrücken [...]. Mit 23,5 Prozent folgen W-Fragen. ${ }^{32}$

\section{Gespräch zwischen der Anästhesistin A101 und dem Patienten P104}

Der Patient (P104) ist ein 33-jähriger Grafiker ohne nennenswerte Operationserfahrung. Er ist kooperativ, interessiert und in Bezug auf die Operation (Knochenbruch des linken Armes) und die damit verbundene Anästhesie besorgt. ${ }^{33}$ Für die Operation stehen zwei mögliche Anästhesieverfahren zur Auswahl: Es könnte eine Allgemeinanästhesie (alltagsspr. Vollnarkose) oder eine axilläre Plexusanästhesie durchgeführt werden. Die axilläre Plexusanästhesie ist eine Form der Regionalanästhesie bei der durch die Injektion eines Lokalanästhetikums im Achselbereich ein Nervengeflecht vorübergehend ausgeschaltet wird und so eine Operation am Arm möglich ist. ${ }^{34}$ Grundsätzlich ist eine Regionalanästhesie mit weniger Risiken verbun- 
den als eine Allgemeinanästhesie und daher in der Regel vorzuziehen. Im Gespräch klärt die Anästhesistin (A101) den Patienten über beide Verfahren auf und legt ihm nahe, sein Einverständnis zu einer Teilnarkose zu geben. Die Dauer des Gespräches beträgt 6 Minuten und 51 Sekunden.

Bereits in der ersten Gesprächsminute informiert die Ärztin P104 darüber, dass zwei Anästhesieverfahren zur Auswahl stehen: »o:ke: herr P104 sie haben eigentlich zwei möglichkeiten als anästhesieverfahren entweder ein teilnarkose das heißt dass den arm nur betäubt wird o:der vollnarkose « (62-68). Durch die Formulierung »sie haben [...] zwei möglichkeiten« stellt die Ärztin die Entscheidung in den Verantwortungsbereich des Patienten. Allerdings hat zu diesem Zeitpunkt noch kein Austausch an entsprechenden Informationen stattgefunden und der Patient sieht sich (noch) nicht in der Lage, eine Entscheidung zu treffen. Stattdessen beginnt der Patient, Fragen zu stellen (im Transkript fett markiert), um sich die Information einzuholen, die er für relevant hält:

[23] Transkript: A101P104pddb ${ }^{35}$

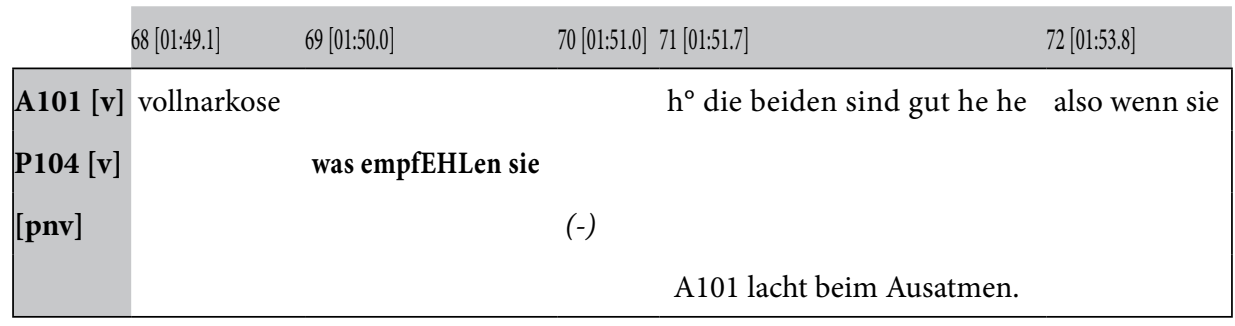

$[24]$

$73[01: 57.4] \quad 74[01: 57.9] 75[01: 58.3]$

\begin{tabular}{llc} 
A101 [v] kooperativ sind (.) dann machen wir gern ein teilnarkose & weil \\
P104 [v] & öh & klar \\
[pnv] & P104 guckt erstaunt und zuckt mit den Schultern. (-) & \\
\hline
\end{tabular}

$[25]$

$76[01: 58.8] 77[01: 59.3]$

78 [02:03.2]

\begin{tabular}{|c|c|}
\hline $\begin{array}{l}\text { A101 [v] eh } \\
\text { P104 [v] }\end{array}$ & das der arm wird eh nur betäubt aber sie sind wach anschpreschbar \\
\hline [pnv] & $\begin{array}{l}\text { P104 faltet mühevoll den Fragebogen, da er seine linke Hand nicht } \\
\text { richtig benutzen kann. }\end{array}$ \\
\hline
\end{tabular}




\begin{tabular}{|lc|}
\multicolumn{1}{ll}{.} & $79[02: 03.6]$ \\
\hline $\begin{array}{l}\text { A101 [v] } \\
\text { P104 [v] hm }\end{array}$ & aber jederzeit können sie natürlich was zum dämma schlafen be kommen \\
[pnv] & A101 macht mit der linken Hand eine Geste. \\
\hline
\end{tabular}

[27]

\begin{tabular}{|lccc|}
\multicolumn{1}{ll}{} & $81[02: 07.2]$ & $82[02: 07.8]$ & $83[02: 08.0]$ \\
\hline $\begin{array}{l}\text { A101 [v] } \\
\text { P104 [v] ja: }\end{array}$ & ja & & äh oder (.) vollnarkose das heißt dass sie tief und fest \\
[pnv] & & (.) & P104 faltet den Fragebogen \\
\hline
\end{tabular}

\section{$84[02: 12.2] \quad 85[02: 12.8]$}

A101 [v] schlafen werden

P104 [v]

[pnv]

[29]

\begin{tabular}{|c|c|c|c|c|}
\hline .. & $86[02: 16.2]$ & $87[02: 16.5]$ & $88[02: 17.6]$ & $89[02: 18.7]$ \\
\hline $\begin{array}{l}\text { A101 [v] } \\
\text { P104 [v] mehr } \\
{[\text { pnv] }}\end{array}$ & $\begin{array}{r}\text { nein } \\
\text { ri siken }\end{array}$ & mit sich als eine & $\begin{array}{l}\text { also jede narkose } \\
\text { teilnarkose }\end{array}$ & also jede medizinische eingriff \\
\hline
\end{tabular}

[30]

\begin{tabular}{|lllll|}
\multicolumn{1}{l|}{} & $90[02: 22.7]$ & $91[02: 23.7]$ & $92[02: 24.5]$ \\
\hline $\begin{array}{l}\text { A101 [v] baar risiken hat hehe das ist genau so } \\
\text { P104 [v] }\end{array}$ & ${ }^{\circ} \mathrm{h}$ also die risiken & steht hier und & auch also \\
& ja klar & hm_hm \\
[pnv] & $\begin{array}{l}\text { P104 lässt den Blick durch den Raum } \\
\text { wandern. }\end{array}$ & & \\
\hline
\end{tabular}

[31]

\begin{tabular}{|c|c|c|c|}
\hline & $93[02: 25.2]$ & $94[02: 27.2]$ & $95[02: 27.4]$ \\
\hline \multicolumn{4}{|c|}{ beiden anästhesieverfahren } \\
\hline P104 [v] & & & o:ke: und gibt es ein verfahren mit mehr \\
\hline [pnv] & & (.) & P104 gestikuliert. \\
\hline
\end{tabular}




\begin{tabular}{|c|c|c|}
\hline.. & $96[02: 32.2]$ & $97[02: 32.8]$ \\
\hline A101 [v] & also & ich finde mit der \\
\hline P104 [v] risiken oder ist es ist es egal welches es nehm ich nehme & $($ ) & \\
\hline
\end{tabular}

\section{$98[02: 36.8] 99[02: 37.1] \quad 100[02: 37.5]$}

\begin{tabular}{|c|c|c|}
\hline P104 [v] & o:ke: & gut \\
\hline [pnv] & (.) & \\
\hline
\end{tabular}

\section{$102[02: 38.8] \quad 103[02: 39.3] \quad 104[02: 39.7]$}

\begin{tabular}{|c|c|c|}
\hline A101 [v] & definitiv besser & \\
\hline P104 [v] & ehm & geht das so dass ich ein bisschen was kriege damit ich ein \\
\hline$[\mathbf{p n v}]$ & & P104 gestikuliert mit dem gesunden Arm und wippt \\
\hline
\end{tabular}

\begin{tabular}{|lllll|}
\multicolumn{1}{l|}{} & $105[02: 42.9]$ & $106[02: 43.5]$ & $107[02: 44.5]$ & $108[02: 45.2]$ \\
\hline A101 [v] & sicher & & sicher \\
P104 [v] bisschen: & duselig bin & und dann erst & öh die & \\
[pnv] den Kopfnach rechts und links. & A101 nickt. & & P104 deutet auf seine linke Achselhöhle. \\
\hline
\end{tabular}

[36]

\begin{tabular}{|lllll|}
\multicolumn{1}{c}{$109[02: 45.7]$} & $110[02: 46.5]$ & $111[02: 47.1]$ & $112[02: 47.6]$ \\
\hline A101 [v] & das ist klar & ja & o:ke: also wie läuft das ab $\uparrow$ ich erkläre es \\
P104 [v] teilnarkose & dann mache ich das & so & \\
[pnv] & A101 nickt. & & & A101 schlägt den Fragebogen auf und dreht ihn P104 hin. P104 \\
\hline
\end{tabular}

Der Patient beginnt mit einer expliziten Bitte um Empfehlung: "was empfEHLen sie« (69). Die Ärztin antwortet: »die beide sind gut« (71). Damit kommt sie der Bitte des Patienten nicht nach und gibt auch keine Information, die ihm bei der Entscheidungsfindung helfen könnte. Im Folgenden informiert sie: "also wenn sie kooperativ sind (.) dann machen wir gern ein teilnarkose« (72). Mit »wir« bezieht die Ärztin sich entweder auf das 
Operationsteam oder auf die Klinik allgemein und informiert, dass dieses >wir< eine Regionalanästhesie vorzieht. Die Ärztin begründet dies mit: »weil eh das der arm wird eh nur betäubt aber sie sind wach anschpreschbar« (75-77). A101 informiert hier darüber, welche Vorzüge eine Regionalanästhesie für das medizinische Personal hat, nicht aber, welches Verfahren für den Patienten das bessere wäre. Dabei fügt sie die eher unangemessene Einschränkung hinzu: »wenn sie kooperativ sind « (72). Diese Information und Einschränkung lösen bei dem Patienten sichtlich Verwirrung aus: Er zuckt mit den Achseln, guckt erstaunt, zögert und sagt: »öh klar« (74-75). Die Irritation des Patienten ist nachvollziehbar, da A101 der Bitte des Patienten nicht nachkommt und durch ihre Einschränkung die Möglichkeit impliziert, dass sich P104 unkooperativ verhalten könnte.

Die Ärztin fährt fort mit: »aber jederzeit können sie natürlich was zum dämma schlafen bekommen « (79). Hier informiert die Ärztin, dass der Patient bei einer Regionalanästhesie zusätzlich ein Beruhigungsmittel bekommen kann. Unklar bleibt, wann (jederzeit, vor oder auch während der Operation?) und in welcher Form dem Patienten ein solches Mittel verabreicht werden kann. Unklar bleibt auch, um welches Mittel es sich handelt und welche Wirkung es im Einzelnen hat. Weiter erklärt die Ärztin: "äh oder (.) vollnarkose das heißt dass sie tief und fest schlafen werden « (83). Es wird nicht klar welche Möglichkeiten durch die Konjunktion `oder` gegenübergestellt werden. Einerseits könnte gemeint sein, dass der Patient während einer Regionalanästhesie zusätzlich ein Beruhigungsmittel bekommen oder nachträglich eine Allgemeinanästhesie eingeleitet werden kann. Andererseits könnte gemeint sein, dass der Patient entweder eine Regionalanästhesie (und ggf. ein zusätzliches Beruhigungsmittel) oder eine Allgemeinanästhesie bekommen kann.

Da der Patient noch keine Information bekommen hat, die ihm bei der Entscheidung für eines der beiden Verfahren hilfreich erscheint, konkretisiert er seine Bitte: »ehm bringt denn eine vollnarkose irgendwie mehr mehr risiken mit sich als eine teilnarkose« (85-88). Mit seiner Frage greift der Patient einen konkreten Aspekt heraus, der seiner Meinung nach relevant für die Entscheidung ist - die mit den Verfahren verbundenen Risiken.

Auf die Frage antwortet A101, noch während P104 spricht mit »nein« (86). Es kann davon ausgegangen werden, dass die Ärztin die Frage nicht (vollständig) verstanden hat, da außer Frage steht, dass eine Allgemeinanästhesie mit mehr Risiken verbunden ist als eine Regionalanästhesie. Im Folgenden erklärt die Ärztin: »also jede narkose also jede medizinische eingriff baar risiken hat« (88-89). Wieder geht die Ärztin nicht auf die Bitte des Patienten ein, sondern reproduziert eine Standardformulierung zur Relativierung von Risiken, die sie in jedem Aufklärungsgespräch verwendet. Das folgende kurze 
Lachen der Ärztin könnte ein Versuch sein, die Situation etwas zu entspannen. Der Patient reagiert darauf nicht, sondern lässt seinen Blick durch den Raum wandern. Nun fügt die Ärztin hinzu: »also die risiken steht hier und auch also in beide anästhesieverfahren « (90-93). Hier verweist sie auf den Aufklärungsbogen und die darin enthaltene Information über die Risiken verschiedener Anästhesieverfahren. Damit lagert sie das Thema aus dem Gespräch aus und überlässt es dem Patienten, dem sechsseitigen Bogen die für ihn relevanten Informationen zu entnehmen und abzuwägen. Der Patient zögert kurz und reformuliert dann seine Frage: "gibt es ein verfahren mit mehr risiken oder ist es egal welches es nehm ich nehme« (95). Diese Reformulierung lässt darauf schließen, dass der Patient annimmt, dass die Ärztin seine Frage nicht verstanden hat. Er verhält sich als kompetenter Sprecher, ${ }^{36}$ indem er versucht, durch eine Reformulierung Verstehen herzustellen.

Erst jetzt beantwortet A101 die Frage des Patienten: »also ich finde mit der mit der eh vollnarkose ist eher mehr risiken aber mit teilnarkose ist definitiv besser « (96-103). Durch die Verwendung von >ich finde` markiert die Ärztin ihre Antwort als persönliche Meinung und durch >eher` relativiert sie ihre Einschätzung. Beides ist im Kontext eines anästhesiologischen Aufklärungsgespräches eher unangemessen. Angemessener wäre eine objektive Gegenüberstellung der Risiken beider Verfahren. Im weiteren Verlauf des Gespräches (siehe unten) wird deutlich, dass die Ärztin einem bestimmten Gesprächsablauf folgt, bei dem die Risikoaufklärung erst zu einem späteren Zeitpunkt eingeplant ist. Der Ärztin scheint es nicht zu gelingen, spontan von diesem Ablauf abzuweichen. Dasselbe zeigt sich auch in allen weiteren Aufklärungsgesprächen dieser Ärztin sowie in den Gesprächen der anderen Anästhesisten/innen, die an der Studie teilgenommen haben. ${ }^{37}$

Im Folgenden wird deutlich, dass der Patient die Empfehlung der Ärztin annimmt. Allerdings hat er nun noch eine Frage zum bevorzugten Anästhesieverfahren: "geht das so dass ich ein bisschen was kriege damit ich ein bisschen: duselig bin und dann erst öh die teilnarkose« (104-109). Es fällt auf, dass der Patient durch Körpersprache zusätzlich versucht Verstehen zu sichern: Er wippt den Kopf hin und her, um »duselig« zu veranschaulichen und bei »teilnarkose« deutet er auf seine linke Achselhöhle. Auch hier verhält er sich als kompetenter Sprecher und versucht durch nonverbale Kommunikation zusätzlich Verstehen zu sichern. Seine Frage offenbart, dass der Patient die ärztliche Information in den Segmenten 79 bis 83 nicht verstanden hat. Es liegt nahe, dies auf die oben beschriebene Uneindeutigkeit 
der Äußerung von A101 an dieser Stelle zurückzuführen. Darüber hinaus deutet die Frage darauf hin, dass der Patient mit der üblichen Angst vor eine Operation beschäftigt ist. Nachdem die Ärztin ihm mehrfach versichert hat, dass er ein entsprechendes Medikament bekommen kann (105-109), entscheidet P104: »dann mache ich das so« (110-111).

Nun informiert die Ärztin den Patienten über den Ablauf einer axillären Plexusanästhesie:

o:ke: also wie läuft das ab $\uparrow$ ich erkläre es ihnen über (.) das bild das ist hier in de aktik wo eh da liegt die nerven die den den ganzen arm versorgt mit hilfe unser ultraschallsgerät kann man genau definieren wo die nerven sind (.) und wenn wir die nerven erwischen wird_n lokalbetäubung gespritzt (112-120).

Bei dieser Information fallen die ungünstige Wortwahl und mehrere sprachliche Fehler auf. Die Formulierung »wenn wir die nerven erwischen« kann eine beunruhigende Wirkung haben. Darüber hinaus können die sprachlichen Fehler - beispielsweise "aktik « statt >Achsel - das Verstehen beeinträchtigen und zusätzlich beunruhigen. An dieser Stelle soll darauf hingewiesen werden, dass die Beschreibung des Ablaufs einer axillären Plexusanästhesie eine Standardinformation in diesem Gesprächstyp ist. Solche Standardinformationen werden von den ausländischen Ärzten/innen selbst als sprachlich wenig herausfordernd beschrieben, da sie im Vorfeld auswendig gelernt und lediglich reproduziert werden müssen. ${ }^{38}$ Dennoch enthält die Äußerung der Ärztin eine Reihe von sprachlichen Fehlern. Die nun folgende Frage des Patienten deutet darauf hin, dass er tatsächlich beunruhigt ist:

[40] Transkript: A101P104pddb ${ }^{39}$

\begin{tabular}{|l|lccc|}
\multicolumn{1}{ll}{$.120[03: 06.3]$} & $121[03: 08.1]$ & $122[03: 08.8]$ & $123[03: 09.2]$ \\
\hline A101 [v] & wird_n lokalbetäubung gespritzt & & wann & \\
P104 [v] & super & wird & wird das zuerst öh irgendwie \\
[pnv] & & & & P104 deutet auf die Abbildung. \\
\hline
\end{tabular}

[41]

\begin{tabular}{|c|c|c|c|c|}
\hline & $124[03: 10.8]$ & $125[03: 11.7]$ & $126[03: 12.1]$ & $127[03: 13.0]$ \\
\hline A101 [v] & wird das gemacht & & genau bevor sie & überhaupt in o:pe: reinfahren \\
\hline P104 [v] & angetäubt & bevor & ah o:ke: & \\
\hline [pnv] & P104 deutet auf seine linke Achselhöhle. & & & \\
\hline
\end{tabular}


Der Patient unterbricht die Ärztin mit der Frage »wird das zuerst öh irgendwie angetäubt«(123-124). Auch hier zieht der Patient nonverbale Kommunikation zur Verstehenssicherung heran, indem er auf die Abbildung im Aufklärungsbogen und auch wieder auf seine Achselhöhle deutet. Darüber hinaus scheint er um Worte zu ringen (»öh irgendwie«) und letztlich erfindet er das Wort »angetäubt«. Die Frage des Patienten lässt auf seine Beunruhigung schließen. Er scheint davon auszugehen, dass die Injektion des Regionalanästhetikums in die Achselhöhle schmerzhaft ist. Die Ärztin antwortet an dieser Stelle: "genau bevor sie überhaupt in o:pe: reinfahren « (126-127). Im weiteren Verlauf des Gespräches wird deutlich, dass der Patient diese Antwort als Bestätigung seiner Annahme versteht, dass er vor der Injektion des Regionalanästhetikums eine leichtere Betäubung injiziert bekommt. Unten wird ausgeführt, dass hier ein Missverständnis vorliegt und die Ärztin sich lediglich auf eine Beruhigungstablette bezieht, die der Patient im Vorfeld einnehmen kann. Nun folgt eine Beschreibung des weiteren Ablaufs am Operationstag durch die Ärztin:

[42] Transkript: A101P104pddb ${ }^{40}$

\begin{tabular}{|lclll|}
\multicolumn{1}{ll}{$128[03: 15.0]$} & $129[03: 15.6]$ & $130[03: 16.3]$ & $131[03: 18.8]$ & $132[03: 19.3]$ \\
\hline A101 [v] & o:ke: $\uparrow$ & und ehm wenn de äh die be & täubung sitzt & dann (.) kann man \\
P104 [v] o:ke: & o:ke: & & hm_hm & \\
\hline
\end{tabular}

\section{$133[03: 23.1] \quad 134[03: 23.4] \quad 135[03: 23.8]$}

A101 [v] anfangen (.) dauert nur ein paar minuten und der arm bleibt betäu äh taub
P104 [v] $[44]$

\begin{tabular}{|llll|}
\multicolumn{1}{c|}{137 [03:26.4] } & $138[03: 28.4]$ & $139[03: 30.4]$ & \\
\hline A101 [v] vielleicht für EIN halbe stunde & das reicht vollkommen aus für die & & äh für \\
P104 [v] & & für & \\
& Handy klingelt. & Handy klingelt. & Handy \\
\hline
\end{tabular}

$[45]$

\begin{tabular}{|llll|}
\multicolumn{1}{|l}{.} & $141[03: 32.0]$ & $142[03: 32.5]$ & $143[03: 34.1]$ \\
\hline A101 [v] die opera & & ein ein und_a halb also & neunzig minuten \\
P104 [v] $\begin{array}{l}\text { anderthalb } \uparrow \text { oder eine halbe } \\
\text { klingelt. }\end{array}$ & stunde & & also neunzig \\
Handy klingelt. & Handy klingelt. & Handy klingelt \\
\hline
\end{tabular}


[46]

\begin{tabular}{|lllll|}
\multicolumn{1}{ll}{..} & $144[03: 35.1]$ & $145[03: 36.2]$ & $146[03: 36.9]$ & $147[03: 37.9]$ \\
\hline A101 [v] & & entschuldigen sie & \\
P104 [v] minuten & ah o:ke: hmhm hmhm & kein problem & \\
[pnv] & & & \multicolumn{2}{c|}{ A101 nimmt das } \\
& Handy klingelt & Handy klingelt & Handy klingelt & Handy klingelt \\
\hline
\end{tabular}

[47]

\begin{tabular}{|c|c|c|}
\hline &. & $148[03: 42.6]$ \\
\hline A101 [v] & & hallo $\uparrow$ entschuldigung ich kann jetzt nicht ich melde mich (.) äh später \\
\hline [pnv] & Gespräch an. & A101 telefoniert. \\
\hline
\end{tabular}

Bei dieser Beschreibung sagt die Ärztin »EIN halbe stunde« (137) statt "anderthalb Stunden «. Dieser sprachliche Fehler führt dazu, dass der Patient die Zeitangabe nicht versteht und nachfragt: "anderthalb $\uparrow$ oder eine halbe stunde« (140-141). Zunächst reagiert die Ärztin mit einer noch immer fehlerhaften Selbstkorrektur »ein ein und_a halb« (142) und fügt dann die Reformulierung "also neunzig minuten « (142-143) hinzu. Während der Patient nun Verstehen signalisiert, klingelt das Arbeitshandy der Ärztin und das Gespräch wird durch ein Telefonat unterbrochen.

Der Patient nutzt die Unterbrechung, um ein weiteres Verfahren der Verstehenssicherung einzusetzen - das Zusammenfassen bzw. Wiedergeben in eigenen Worten - wodurch sich einmal mehr zeigt, dass der Patient unsicher ist, ob er die Ausführungen der Ärztin verstanden hat, und dass er es für notwendig hält, Verstehen zu sichern.

[48] Transkript: A101P104pddb ${ }^{41}$

\begin{tabular}{|c|c|c|c|c|c|}
\hline & $149[03: 46.6] 150[03: 47.6]$ & 151 [03:48.3] & $152[03: 49.7] 153[03: 50.5]$ & $154[03: 50.9]$ & $155[03: 51.3]$ \\
\hline A101 [v] & tschüss & & & ja $\uparrow$ & \\
\hline P104 [v] & & & o:ke: & also kriege & ich \\
\hline$[\mathrm{pnv}]$ & $(-)$ & A101 steckt das Telefon in die Kitteltasche. & $(-)$ & & P104 \\
\hline
\end{tabular}

[49]

P104 [v] erstmal denk denke ich irgendwo eine kleine spritze und dann wird das taub und [pnv] greift sich an die linke Schulter. 


\begin{tabular}{|llll|}
\multicolumn{1}{l|}{} & $156[03: 55.7]$ & $157[03: 57.1]$ & $158[03: 57.6]$ \\
\hline A101 [v] & ne: es ist nur eine spritze & hier (.) \\
P104 [v] dann kriege ich die große spritze hier & & ach so das & ist nur \\
[pnv] mit dem Zeigefinger eine Spritze in die Achselhöhle an. & & A101 deutet \\
\hline
\end{tabular}

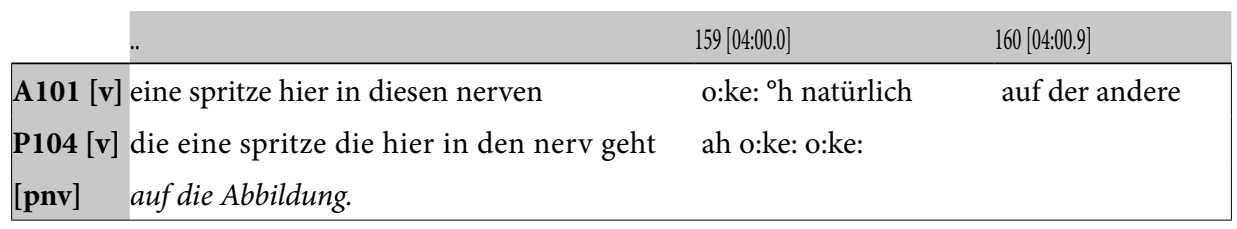

Dank der Zusammenfassung des Patienten tritt zutage, dass ein Missverständnis vorliegt: Wie oben bereits beschrieben, geht der Patient davon aus, dass er zunächst eine leichte Betäubung (»also kriege ich erstmal denk ich irgendwo eine kleine spritze und dann wird das taub «(154-155)) und erst im Anschluss die Regionalanästhesie (»und dann kriege ich die große spritze hier (155)) injiziert bekommt. Diese Ausführung sowie die Unterscheidung »kleine spritze« und "große spritze« weisen erneut auf die Annahme des Patienten hin, dass das Spritzen der Regionalanästhesie schmerzhaft ist. Die Ärztin unterbricht den Patienten an dieser Stelle mit einem Widerspruch (156) und gibt die folgende Information in Bezug auf die zum Einsatz kommenden Spritzen:

hier (.) eine spritze hier in diesen nerven o:ke: ${ }^{\circ} \mathrm{h}$ natürlich auf der andere seite we äh kriegen sie ein venöse zuGANG zum flüssigkeit äh äh zu bekommen äh antibiotikum zuSÄTZliche schmerz mittel da ist klar also venöse zuGANG muss man sowieso kriegen (158-166).

Es gelingt der Ärztin hier klarzustellen, dass es nur eine Injektion und darüber hinaus lediglich einen venösen Zugang an der anderen Hand geben wird. Der Patient signalisiert nun durch eine Wiederholung Verstehen: »ach so das ist nur die eine spritze die hier in den nerv geht « (158). Es muss an dieser Stelle festgestellt werden, dass das Missverständnis nur durch die vom Patienten initiierte Verstehenssicherung bearbeitet werden konnte. Durch die Klärung des Missverständnisses steht der Patient nun allerdings wieder vor dem Problem, dass er Angst vor Schmerzen bei der Injektion des Anästhetikums hat. Die Ausführungen der Ärztin konnten ihm diese Angst bisher nicht nehmen, wahrscheinlich haben sie eher zusätzlich zu seiner Beunruhigung beigetragen. Dementsprechend stellt der Patient die folgende Frage: 
[54] Transkript: A101P104pddb ${ }^{42}$

\begin{tabular}{|llll|}
\multicolumn{1}{c|}{$166[04: 11.5]$} & $167[04: 13.6]$ & $168[04: 14.2]$ \\
\hline A101 [v] venöse & zuGANG muss man sowieso kriegen & ${ }^{\circ} \mathrm{h}$ & \\
P104 [v] hmhm & & o:ke: ist das denn sehr \\
[pnv] & & $(-)$ & P104 zeigt auf seine Achselhöhle \\
\hline
\end{tabular}

\begin{tabular}{|c|c|c|c|}
\hline. & $169[04: 16.2]$ & $170[04: 17.6]$ & $171[04: 18.6]$ \\
\hline A101 [v] & nein wir machen das unter & lokalbetäubung & also (.) \\
\hline $\begin{array}{l}\text { P104 [v] schmerzhaft diese spritze hier } \uparrow \\
\text { [pnv] }\end{array}$ & in den nerv $\uparrow$ & & ah ah ah \\
\hline
\end{tabular}

[56]

$172[04: 20.5]$

A101 [v] jetzt is so einen klein pieks genau so wie beim zahnarzt P104 [v] o:ke: o:ke:

\section{3[04:22.4] $174[04: 22.8] \quad 175[04: 23.3]$}

und dann geht es

hmhm hm hm

[57]

\begin{tabular}{|lllllll|}
\multicolumn{1}{ll}{..} & $176[04: 24.0]$ & $177[04: 24.7]$ & $178[04: 25.1]$ & $179[04: 25.5]$ & 180 [04:26.0] & 181 [04:26.6] \\
\hline A101 [v] los & & & ja $\uparrow$ & alles klar & sie kommen auch an dem tag \\
P104 [v] & o:ke: gut & & & ja & \\
[pnv] & & $(-)$ & & & $(-)$ & \\
\hline
\end{tabular}

An dieser Stelle formuliert der Patient den Grund seiner Besorgnis explizit: »ist das denn sehr schmerzhaft« (168). In seiner Konkretisierung »diese spritze hier $\uparrow$ in den nerv $\uparrow$ « nimmt er wieder Bezug auf ungünstig formulierte Information der Ärztin (112-120). Die Antwort der Ärztin »nein wir machen das unter lokalbetäubung " (169) ist irritierend. Als Antwort auf die Frage des Patienten kann diese Äußerung nur wie folgt verstanden werden: "nein «, d.h. das Injizieren des Regionalanästhetikums ist nicht schmerzhaft und »wir machen das unter lokalbetäubung «, d.h. das Injizieren findet unter Lokalbetäubung statt. Hier scheint sich das Missverständnis von oben zu wiederholen. Die Äußerung der Ärztin kann hier nur zusätzliche Verwirrung, nicht aber Beruhigung bewirken. Die folgende Erläuterung der Ärztin »is so einen klein pieks genau so wie beim zahnarzt« (171-172) kann ebenfalls beunruhigend wirken. Die Beschreibung »klein pieks« könnte beruhigend wirken, wenn nicht der äußerst ungünstige Vergleich 
»wie beim zahnarzt« (172) folgen würde. Der Patient scheint sich hier nun gewissermaßen seinem Schicksal zu ergeben, und die Ärztin fährt mit dem Gespräch fort:

[58] Transkript: A101P104pddb ${ }^{43}$

\begin{tabular}{|c|c|c|c|c|}
\hline & .. & 182 [04:28.6] & $183[04: 30.7]$ & $184[04: 31.6]$ \\
\hline A101 [v] & nüchtern & also das ist auch sehr wichtig & & öhh $^{\circ}$ soll ihnen jetzt erzählen über \\
\hline P104 [v] & & ja klar (.) hm hm & & \\
\hline [pnv] & & A101 blättert im Fragebogen & $(-)$ & \\
\hline
\end{tabular}

[59]

\begin{tabular}{|llc|}
\multicolumn{1}{l|}{.} & $185[04: 35.9]$ & $186[04: 37.5]$ \\
\hline A101 [v] die risiken auch von diesem narkose & also jeder medizinische eingriff & äh besser \\
P104 [v] & nä: besser \\
\hline
\end{tabular}

$[60]$

\begin{tabular}{|c|c|c|c|}
\hline &. & $187[04: 39.6]$ & 188 [04:41.4] \\
\hline A101 [v] & nicht wollen sie nicht & & \\
\hline P104 [v] & nicht (.) ich habe schon & mal was gelesen das ist klar also & schiefgehen kann es ja \\
\hline
\end{tabular}

$[61]$

\begin{tabular}{|lll|}
\multicolumn{1}{l|}{} & $189[04: 43.6]$ & $190[04: 45.6]$ \\
\hline $\begin{array}{l}\text { P104 [v] immer } \\
\text { [pnv] }\end{array}$ & aber $\mathrm{h}^{\circ}$ äh ich denke bei der lokalen narkose ist das ist das \\
\hline
\end{tabular}

\begin{tabular}{|llcl|}
\multicolumn{1}{l|}{ 191 [04:49.0] } & $192[04: 51.3]$ & $193[04: 55.1]$ \\
\hline A101 [v] & ich finde es auch noch geringer das ist klar & \\
P104 [v] & risiko noch & geringer (.) ja: gut & ehm: ist das \\
[pnv] & & A101 schreibt. & A101 schreibt. \\
\hline
\end{tabular}

Zunächst gibt die Ärztin P104 die Anweisung, am Operationstag nüchtern (d.h. mit leerem Magen) zu erscheinen. Dann fragt sie, ob der Patient die Risiken der Narkose von ihr hören möchte: »soll ihnen jetzt erzählen über die risiken auch von diesem narkose« (184). Mit »jetzt« weist sie darauf hin, dass dies der für die Risikoaufklärung vorgesehene Zeitpunkt im Gespräch ist. Wie oben bereits beschrieben folgt sie damit ihrem üblichen 
Gesprächsablauf - einem Ablauf, der sich eher für Gespräche eignet, in denen nur ein Anästhesieverfahren in Frage kommt. Im Gespräch mit P104 wirkt die Frage zur Risikoaufklärung besonders irritierend, da der Patient zuvor nach Risiken gefragt und keine Information von der Ärztin bekommen hat. Da der Patient nicht antwortet, fährt die Ärztin mit derselben Standardformulierung zur Relativierung von Risiken fort, die sie bereits in Segment 89 verwendet hat. Nun unterbricht der Patient die Ärztin mit »nä: besser nicht« (186). Für den Patienten wäre eine Information über die Risiken der Regionalanästhesie im Vergleich zur Allgemeinanästhesie für die Entscheidung relevant gewesen, in welches der Verfahren er einwilligen möchte. Da er sich nun bereits mündlich mit der axillären Plexusanästhesie einverstanden erklärt hat (siehe 110), kann die Risikoaufklärung ihn an dieser Stelle nur noch weiter beunruhigen. P104 erklärt vage, er habe »Schon mal was gelesen « (186-187) und fügt sein Fazit hinzu »das ist klar also schiefgehen kann es ja immer (187-188). Nun kommt der Patient noch einmal auf den Kontext zurück, in dem die Frage nach den Risiken für ihn von Bedeutung war: »ich denke bei der lokalen narkose ist das ist das risiko noch geringer « (190). Der Patient wiederholt noch einmal, wie er die Information der Ärztin in den Segmenten 96 bis 102 verstanden hat. Daraus wird deutlich, dass er noch unsicher war, ob er die Ärztin richtig verstanden hatte. Der Patient möchte an dieser Stelle noch einmal sicherstellen, dass die Regionalanästhesie tatsächlich das weniger risikoreiche Verfahren ist. Die Ärztin bestätigt dieses Verständnis. Dabei markiert sie ihre Bestätigung wiederum durch »ich finde « als persönliche Meinung (vgl. 97), was in diesem beruflichen Kontext unangemessen ist. Nun stellt der Patient weitere Fragen zum Ablauf und zur Wirkung der Plexusanästhesie:

[63] Transkript: A101P104pddb ${ }^{44}$

P104 [v] sehr laut eigentlich die operation $\uparrow$ also kriege ich da viel mit von irgendwie mit [pnv]

[64]

\begin{tabular}{|llll|}
\multicolumn{1}{l|}{} & 194 [05:01.9] & 195[05:03.9] & 196[05:05.7] \\
\hline A101 [v] & nein (.) das nicht auch & & \\
P104 [v] bohren und sägen oder irgendwas $\uparrow$ & oder o:ke: gut o:ke & \multicolumn{1}{c|}{ und falls } \\
[pnv] & A101 schüttelt den Kopf. & A101 schreibt. & \\
\hline
\end{tabular}


[65]

\begin{tabular}{|ll|}
\multicolumn{1}{|l}{ A101 [v] } & $197[05: 09.1]$ \\
P104 [v] ich dann während der operation sage o:ke: ich spüre irgendwas oder & ehm ehm \\
\hline
\end{tabular}

[66]

\begin{tabular}{|c|c|c|c|}
\hline & $198[05: 10.0]$ & $199[05: 11.1]$ & $201[05: 13.0]$ \\
\hline A101 [v] & an ihrer seite immer anästhe & sist also sie sin nisch alleine & $\mathrm{ehh}^{\circ}$ \\
\hline \multirow[t]{2}{*}{ P104 [v] } & & also dann könnten sie noch noch & ein bisschen noch \\
\hline & & & P104 lacht. \\
\hline
\end{tabular}

[67]

\begin{tabular}{|c|c|c|c|c|}
\hline & $202[05: 13.7]$ & $203[05: 14.5]$ & $204[05: 15.5]$ & $205[05: 17.0]$ \\
\hline A101 [v] & \multicolumn{4}{|c|}{ dann kann man auch ab jetzt schreiben } \\
\hline P104 [v] & \multicolumn{3}{|c|}{ ein bisschen heh mehr betäuben und dann heheh ${ }^{\circ}$} & ja \\
\hline & A101 lacht beim Ausatmen. & & A101 schreibt. & \\
\hline
\end{tabular}

[68]

\begin{tabular}{|c|c|c|c|c|c|}
\hline & $206[05: 17.7]$ & $207[05: 19.8]$ & $208[05: 20.4]$ & $209[05: 23.0]$ & 210 [05:23.3] \\
\hline A101 [v] & & & also bevor sie äh ins o:pe: gebracht werden & & äh wird äh \\
\hline $\begin{array}{l}\text { P104 [v] } \\
{[\text { pnv] }}\end{array}$ & $(--)$ & ( ) & & $\mathrm{hmhm}$ & \\
\hline
\end{tabular}

[69]

\begin{tabular}{|c|c|c|c|c|}
\hline &.. & $211[05: 27.0]$ & 212 [05:27.4] & $213[05: 28.0]$ \\
\hline A101 [v] & kriegen sie so ein bruhigungsmittel auf station & & erstmal ein & tablette \\
\hline P104 [v] & & ah o:ke: & sehr gut & \\
\hline
\end{tabular}

[70]

\begin{tabular}{|lllll|}
\multicolumn{1}{ll}{} & $214[05: 30.7]$ & $215[05: 31.1]$ & $216[05: 32.0]$ & $217[05: 33.9]$ \\
\hline A101 [v] und noch was äh im o:pe: zusätzlich & & & & \\
P104 [v] & & ah o:ke: gut & & also bin \\
[pnv] & (-) A101 schreibt & & (--) A101 schreibt & A101 schreibt \\
\hline
\end{tabular}

[71]

\begin{tabular}{|lll|}
\multicolumn{1}{l}{$218[05: 35.9]$} & $219[05: 45.3]$ \\
\hline A101 [v] & noch fragen daZU $\uparrow$ \\
P104 [v] & ich jetzt nicht hellwach die ganze zeit & \\
[pnv] & (9.42) P104 schnieft, A101 schreibt. & A101 schaut P104 nicht an.
\end{tabular}


Die Fragen in diesem Ausschnitt zeigen, dass die Ärztin das Ziel den Patienten zu beruhigen, bisher nicht erreicht hat. Der Patient kann sich noch nicht vorstellen, in welchem Zustand er während der Operation sein wird. Er ist besorgt, dass er Schmerzen haben und viel von der Operation mitbekommen könnte. Zunächst fragt er, ob er laute Operationsgeräusche hören wird: »ehm: ist das sehr laut eigentlich die operation $\uparrow$ also kriege ich da viel mit von irgendwie mit bohren und sägen oder irgendwas $\uparrow$ « (193). Die Ärztin verneint diese Frage mit »nein (.) das nicht auch « (194). Es kann nicht nachvollzogen werden, ob sauch $\prec$ hier als Adverb oder Partikel verwendet und was dadurch kommuniziert werden soll. Nun setzt der Patient an, eine weitere Frage zu stellen. Er skizziert den Fall, dass die Anästhesie nicht stark genug wirkt: »und falls ich dann während der operation sage o:ke: ich spüre irgendwas oder « (196). An dieser Stelle wird der Patient von der Ärztin unterbrochen, bevor er eine Frage stellen kann. Die Ärztin versichert ihm, dass er während der Operation nicht alleine ist: »also ich/es stehe an ihrer seite immer anästhesist also sie sin nisch alleine" (197-199). Die Ärztin nimmt offensichtlich die Besorgnis des Patienten wahr und versucht ihn mit der Information, ein/e Anästhesist/in sei während der Operation im Operationssaal anwesend, zu beruhigen. Die folgende Frage des Patienten zeigt, dass die Anwesenheit eines/einer Anästhesisten/in allein ihn noch nicht beruhigt. Er fährt fort, indem er eine Deklarativsatzfrage stellt: »also dann könnten sie noch noch ein bisschen noch ein bisschen heh mehr betäuben « (199-202). Offensichtlich ist der Patient auch besorgt, dass er während der Operation Schmerzen haben könnte. Die Ärztin antwortet auf diese Frage nicht. Stattdessen sagt sie: »dann kann man auch ab jetzt schreiben« (203-204). Gleichzeitig schreibt sie »Dormicum Tab« in das Anästhesieprotokoll. ${ }^{45}$ Dormicum ist der handelsübliche Name eines häufig zur Prämedikation eingesetzten Benzodiazepins, d.h. einer angstlösenden Substanz. ${ }^{46}$ Im Folgenden erklärt sie: »äh wird äh kriegen sie so ein beruhigungsmittel auf station erstmal ein tablette und noch was äh im o:pe: zusätzlich« (210-213). Sie informiert hier den Patienten über die Prämedikation, die sie in das Protokoll eingetragen hat. Unklar bleibt, was der Patient im Operationssaal noch »zusätzlich« (213) bekommt. Möglicherweise bezieht sie sich hier auf die Plexusanästhesie. Allerdings könnte auch etwas anderes gemeint sein.

Die folgende Äußerung des Patienten zeigt, dass er unsicher ist, ob er die für ihn relevanten Informationen verstanden hat. Er versucht, sich noch

46 Vgl. Striebel: Anästhesie - Intensivmedizin - Notfallmedizin, S. 6. 
einmal Verstehen zu sichern, indem er eine Deklarativsatzfrage formuliert: »also bin ich jetzt nicht hellwach die ganze zeit« (217). Wiederum reagiert die Ärztin nicht auf diesen Versuch Verstehen zu sichern. Es fällt auf, dass die Ärztin in diesem kurzen Gesprächsausschnitt (198-219) zweimal auf eine Deklarativsatzfrage nicht reagiert. Die naheliegende Erklärung für dieses Verhalten ist, dass die Ärztin Deklarativsatzfragen nicht als Fragen erkennt und damit auch keine konditionelle Relevanz einer Antwort wahrnimmt. Dieses fehlende sprachliche Wissen ist allerdings für anästhesiologische Aufklärungsgespräche von beachtlicher Relevanz, da - wie oben bereits ausgeführt wurde - Deklarativsatzfragen die häufigste Form von Patientenfragen sind.

Nachdem die Ärztin - wie beschrieben - nicht auf die Frage des Patienten reagiert, schreibt sie ca. 10 Sekunden in den Unterlagen und geht dann zur abschließenden Nachfragemöglichkeit über: "noch fragen daZU $\uparrow$ « (219). Während die Ärztin diese Frage stellt, schaut sie den Patienten nicht an, sondern schreibt weiter in den Unterlagen. Der fehlende Blickkontakt und das Schreiben kommunizieren eine Präferenz für die Antwort `nein‘. Auch in anderen Gesprächen von A101 hat diese Frage weniger die Funktion, dem Patienten Raum zum Fragen zu geben, sondern vielmehr die Funktion, das Gespräch abzuschließen. Tatsächlich ist dies die übliche Verwendung der abschließenden Nachfragemöglichkeit in Arzt-Patienten-Interaktionen. ${ }^{47}$ In dem Gespräch zwischen A101 und P104 wirkt die abschließende Nachfragemöglichkeit zusätzlich irritierend, da der Patient gerade eine Frage gestellt hat, die von der Ärztin noch nicht beantwortet wurde. Entsprechend irritiert reagiert der Patient auf diese Nachfragemöglichkeit:

[72] Transkript: A101P104pddb ${ }^{48}$

\begin{tabular}{|c|c|c|c|c|c|}
\hline & $220[05: 466]$ & $221[05: 47.2] 222[05: 48.4]$ & $223[05: 49.3]$ & $224[05: 50.3]$ & $225[05: 51.1]$ \\
\hline P104 [v] & & ${ }^{\circ} \mathrm{h}$ & weiß ich nicht & & öh ist irgendwie: sollte ich irgendwas \\
\hline [pnv] & $(-)$ & $(-)$ & & $(-)$ & A101 schreibt \\
\hline
\end{tabular}

[73]

$226[05: 55.0]$

P104 [v] besonderes bedenken oder öh so wie ich das jetzt verstanden habe kriege ich ne ne [pnv]

Nach auffälligem Zögern (220) und tiefem Einatmen (221) stellt der Patient fest, dass er nicht weiß, ob er noch Fragen hat (223). Nach weiterem 
Zögern (225) stellt er eine Gegenfrage: »sollte ich irgendwas besonderes bedenken « (225). An dieser Stelle wird deutlich, dass auch das Stellen von Fragen ein gewisses Maß an Wissen und Verstehen voraussetzt. Der Patient kann an dieser Stelle keine konkrete Frage formulieren, scheint aber unsicher zu sein, ob er alle relevanten Informationen verstanden hat. Da die Ärztin hierauf nicht reagiert, fährt er fort, indem er ein weiteres $\mathrm{Mal}$ versucht Verstehen zu sichern: Er beginnt zusammenzufassen, wie er die Informationen über den Ablauf der Anästhesie verstanden hat:

[74] Transkript: A101P104pddb ${ }^{49}$

\begin{tabular}{|ll|}
\multicolumn{1}{l}{$226[05: 55.0]$} \\
\hline $\begin{array}{l}\text { P104 [v] besonderes bedenken oder } \\
\text { [pnv] }\end{array}$
\end{tabular}

\begin{tabular}{|llll|}
\multicolumn{1}{ll}{} & $227[05.59 .4]$ & $228[05.599$ & $229[06: 00.4]$ \\
\hline A101 [v] & genau & dann & \\
P104 [v] en beruhigungsmittel vor der o:pe: & dann kriege ich & hier einen tropf mit weiß \\
[pnv] & A101 nickt. & P104 deutet mit der linken Hand auf den \\
\hline
\end{tabular}

[75]

\begin{tabular}{|llll|}
\multicolumn{1}{l|}{} & $230[06: 02.6]$ & $231[06: 04.1]$ \\
\hline A101 [v] & und flüssigkeit & \\
P104 [v] & ich nicht antibiotikum und & vielleicht noch ein leichtes leich & tes beruhigungsmittel \\
[pnv] & rechten Unterarm. & P104 gestikuliert. & \\
\hline
\end{tabular}

[76]

\begin{tabular}{|ll|}
\multicolumn{1}{c|}{$232[06: 05.1]$} & $233[06: 05.8]$ \\
\hline A101 [v] genau & \\
P104 [v] dann kriege ich diese & spritze \\
[pnv] & $\begin{array}{l}\text { P104 hebt den linken Arm und deutet mit der rechten Hand auf } \\
\text { die linke Achselhöhle. }\end{array}$ \\
\hline
\end{tabular}

[77]

\begin{tabular}{|lcccc|}
\multicolumn{1}{l}{$234[06: 06.4]$} & $235[06: 07.2]$ & $236[06: 07.6]$ & $237[06: 09.4]$ & $238[06: 10.0]$ \\
\hline A101 [v] ganz genau & \multicolumn{4}{c|}{ dann } \\
P104 [v] & & dann ist mein arm betäu betäubt und & ich bin auch leicht & betäubt \\
[pnv] & $(-)$ & P104 streckt den linken Arm vor den Körper. & P104 legt \\
\hline
\end{tabular}


[78]

\begin{tabular}{|lll|}
\multicolumn{1}{ll}{} & $239[06: 10.7]$ & $240[06: 11.6]$ \\
\hline A101 [v] & rischtisch & \\
P104 [v] & dann geht es los dann dauert es & dreißig bis \\
[pnv] den Kopf schief und schließt die Augen. & P104 gestikuliert mit der rechten Hand. P104 schaut A101 an. & P104 zieht die \\
\hline
\end{tabular}

[79]

\begin{tabular}{|c|c|c|c|c|c|c|}
\hline & .. & $241[06: 12.8]$ & 242 [06:13.5] & $243[06: 15.0]$ & $244[06: 15.4$ & $245[06: 15.7]$ \\
\hline A101 [v] & & rischtisch & & ja & & ja \\
\hline P104 [v] & vierzig minuten & in im & geplanten zustand könnte & auch länger & dauern & wenn es \\
\hline [pnv] & Augenbrauen hoch. & A101 nickt. & & A101 nickt. & & A101 nickt. \\
\hline
\end{tabular}

[80]

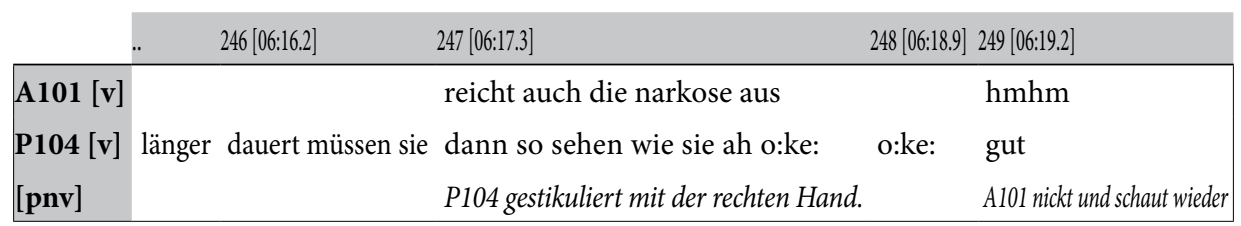

[81]

\begin{tabular}{|c|c|c|c|}
\hline & \multicolumn{2}{|c|}{$250[06: 19.7] 251[06: 20.2]$} & $252[06: 23.2]$ \\
\hline A101 [v] & & & \\
\hline P104 [v] & & und o:ke: ja (.) dann weiß ich ja wie das abläuft & ehm \\
\hline [pnv] & auf die Unterlagen. (- & A101 schreibt & $(--)$ \\
\hline
\end{tabular}

P104 fasst zusammen, dass er zunächst ein Beruhigungsmittel bekommt, dann in den Operationssaal gebracht wird und dort eine Infusion sowie möglicherweise ein weiteres Beruhigungsmittel bekommt. Es bleibt in seiner Schilderung unklar, ob und in welcher Form das zweite Beruhigungsmittel verabreicht wird - wie es auch in der diesbezüglichen Information der Ärztin unklar blieb (210-213). Die Ärztin bestätigt die Ausführungen von P104 und wiederholt ihre Information aus Segment 160, dass über die Infusion nicht nur ein Antibiotikum, sondern auch Flüssigkeit verabreicht wird. P104 schildert weiter, dass er nun das Regionalanästhetikum in die Achselhöhle injiziert bekommt, wodurch sein Arm betäubt wird. Er nimmt an, dass sein übriger Körper auch »leicht betäubt « (237-238) sein wird. Bei seinen Ausführungen fällt auf, dass der Patient zunehmend mehr veranschaulichende Gestik einsetzt. Beispielsweise veranschaulicht er seine Ausführung "ich bin auch leicht betäubt « (237-238) damit, dass er den Kopf schief legt und die Augen schließt (238). Hier scheint er wieder die Rolle 
des kompetenten Sprechers einzunehmen, der versucht, in einem Gespräch mit einem weniger kompetenten Sprecher Verstehen zu ermöglichen.

Im Folgenden schildert der Patient, dass die Operation mindestens 30 Minuten, möglicherweise aber über 40 Minuten dauert. A101 bestätigt die Ausführungen des Patienten. Sie wiederholt nicht die von ihr in Segment 143 angegebene Operationsdauer von 90 Minuten. Möglicherweise möchte sie eine weitere interaktive Aushandlung über die Dauer des Eingriffs vermeiden. Nun skizziert P104 den Fall, dass die Operation länger dauert als geplant (245-246). An dieser Stelle unterbricht A101 den Patienten in seiner Ausführung mit "reicht auch die narkose aus« (247). Erst hier bekommt der Patient die Antwort auf die Deklarativsatzfrage, die er in den Segmenten 199 bis 202 gestellt hat (»also dann könnten sie noch noch ein bisschen noch ein bisschen heh mehr betäuben«). Nun fährt der Patient mit einer konkreten Frage fort:

[82] Transkript: A101P104pddb ${ }^{50}$

\begin{tabular}{|llllll|}
\multicolumn{1}{ll}{$.254[06: 25.7]$} & $255[06: 27.0]$ & $256[06: 28.9]$ & $257[06: 29.4]$ & $258[06: 29.9]$ \\
\hline A101 [v] & & nein & auch nicht & \\
P104 [v] & & kann ich die stelle sehen während ich in & narkose & das wird \\
[pnv] & $(-)$ & P104 streckt den linken Arm vor und legt die Stirn in Falten. & & & \\
\hline
\end{tabular}

\begin{tabular}{|lcccc|}
\multicolumn{1}{l|}{} & $259[06: 30.6]$ & $260[06: 31.2]$ & $261[06: 31.7]$ & $262[06: 32.7]$ \\
\hline A101 [v] & & wird alles & bedeckt & \\
P104 [v] & irgendwie dann & & o:ke: wunderbar & \\
[pnv] & P104 deutet mit der rechten Hand einen Sichtschutz an. & & $(--)$ A101 \\
\hline
\end{tabular}

Der Patient möchte wissen, ob er den operativen Eingriff an seinem Arm sehen kann. Die Ärztin versichert, dass das nicht der Fall sein wird. Sowohl bei seiner Frage als auch bei der Antwort der Ärztin veranschaulicht der Patient die Inhalte wiederum durch auffällige Mimik und Gestik (255 und 259). Während A101 dem Patienten bereits den Bogen zur Unterschrift vorlegt, kommentiert sie in Bezug auf die letzte (möglicherweise auch die vorangegangenen) Fragen des Patienten: 
[84] Transkript: A101P104pddb ${ }^{51}$

\begin{tabular}{|c|c|c|c|c|}
\hline &.. & $263[06: 36.2]$ & $264[06: 38.5]$ & $265[06: 39.0]$ \\
\hline A101 [v] & & ach wir sind nicht so brutal eh ${ }^{\circ}$ hehe & & eh ${ }^{\circ}$ hehe \\
\hline P104 [v] & & & ${ }^{\circ} \mathrm{h} \mathrm{ja}$ & keine \\
\hline [pnv] & dreht den Bogen um, reicht P104 den Kuli. & & & \\
\hline
\end{tabular}

\begin{tabular}{|c|c|c|c|}
\hline & .. & $266[06: 39.7]$ & $267[06: 41.0]$ \\
\hline A101 [v] & & keine ahnung hehe ${ }^{\circ} \mathrm{h}$ & \\
\hline $\begin{array}{l}\text { P104 [v] } \\
{[\text { pnv }]}\end{array}$ & ahnung & äh ich war ich war mit & $\begin{array}{l}\text { sechs zuletzt im eh krankenhaus (.) }{ }^{\circ} \mathrm{h} \text { äh und ich } \\
\text { P104 unterschreibt. }\end{array}$ \\
\hline
\end{tabular}

\begin{tabular}{|lllll|}
\multicolumn{1}{l|}{} & $268[06: 45.9]$ & $269[06: 47.9]$ & $270[06: 48.7]$ & $271[06: 49.1]$ \\
\hline $\begin{array}{l}\text { A101 [v] } \\
\text { P104 [v] }\end{array}$ & weiß gar nicht wie das alles geht & alles klar & vielen dank herr P104 \\
[pnv] & $(-)$ & & $(-)$ & A101 schaut in die Unterlagen. \\
\hline
\end{tabular}

Der Kommentar »ach wir sind nicht so brutal« (263) wertet (zumindest) die vorangegangene Frage des Patienten als absurd. Durch ihr Lachen kommuniziert die Ärztin, dass dieser Kommentar scherzhaft gemeint ist. Möglicherweise möchte sie hierdurch die Situation entspannen oder den Patienten beruhigen. Es fällt allerdings auf, dass der Patient nicht mitlacht. Stattdessen erklärt er, dass er keine Erfahrung mit Operationen hat und keine Kenntnisse über den Ablauf. Es folgt die Verabschiedung.

\section{Schlussfolgerungen}

Das Verhalten des Patienten in dem hier fokussierten Gespräch steht in starkem Kontrast zu den oben dargestellten Ergebnissen der Studie von Klüber et al., ${ }^{52}$ die Aufklärungsgespräche mit Ärzten/innen, die Deutsch als Erstsprache sprechen, in den Blick genommen haben. Der Patient P104 stellt deutlich mehr (insgesamt 11) Fragen als die Patienten/innen in der Studie von Klüber et al. (im Durchschnitt 2,4). Darüber hinaus betreibt der Patient einen hohen interaktiven Aufwand, um Verstehen zu sichern: Er wie-

51 Ebd.

52 Klüber/Motsch/Spranz-Fogasy: Verständigungssicherung. 
derholt die Information der Ärztin, rephrasiert und fasst zusammen. Dabei verwendet er zunehmend mehr veranschaulichende Mimik und Gestik. Es kann davon ausgegangen werden, dass der Patient auf die wahrgenommenen sprachlichen Einschränkungen der Ärztin reagiert. Als kompetenter Sprecher setzt er verschiedene Verfahren der Verstehenssicherung sowie veranschaulichende Mimik/Gestik ein, d.h. er modifiziert den Input, da er Miss-/Nichtverstehen wahrnimmt. ${ }^{53}$

Die Ärztin macht zahlreiche sprachliche Fehler - auch bei Standardformulierungen (z.B. 112-120, 137). Sie verwendet wiederholt unangemessene (z.B. 72, 97, 191) und beunruhigende (z.B. 112-120, 172) Formulierungen. An mehreren Stellen sind ihre Ausführungen unklar (z.B. 79, 83, 194, 208-213), was sich zum Teil auch an den Fragen/Reaktionen des Patienten zeigt (z.B. 85-86, 104-109). Mehrmals treten Missverständnisse nur durch patienteninitiierte Aushandlungen zutage (Anzahl der Injektionen, Dauer der Operation). Immer wieder reagiert die Ärztin auf Fragen des Patienten nicht (z.B. 85-86). Es ist anzunehmen, dass sie Deklarativsatzfragen gar nicht als Frage identifizieren kann (199-217). Die Ärztin orientiert sich stark an ihrem regulären Gesprächsablauf und Standardformulierungen (z.B. 88-89, 185) und hat Schwierigkeiten auf Patienteninitiativen einzugehen, die diesem Ablauf nicht entsprechen. Es ist anzunehmen, dass Ärzte/innen mit Deutsch als Zweitsprache aufgrund von sprachlicher Unsicherheit dazu tendieren, sich stärker an bestimmten Routinen zu orientieren als an ihren Patienten/innen. ${ }^{54}$

Zusammenfassend kann festgehalten werden, dass die eingeschränkten Sprachkompetenzen von A101 ihre Fähigkeit, das Aufklärungsgespräch mit P104 angemessen zu führen, stark beeinträchtigen. Zu demselben Ergebnis führt die Analyse von weiteren anästhesiologischen Aufklärungsgesprächen dieser und zwei weiterer Ärzte/innen mit Deutsch als Zweitsprache. ${ }^{55}$ Alle drei Ärzte/innen haben verschiedene Sprachkurse besucht und erfüllen die in Deutschland vorgegebenen sprachlichen Anforderungen, d.h. sie haben ein B2-Zertifikat vorgelegt und die Fachsprachenprüfung bestanden. Darüber hinaus haben sie zum Zeitpunkt der Datenerhebung seit mindestens drei Jahren an einem deutschen Krankenhaus gearbeitet. Dennoch kommt es in den Gesprächen zu erheblichen sprachlich-kommunikativen Fehlleistungen.

Aus sprachdidaktischer Sicht können folgende Schlüsse gezogen werden: Ausländische Ärzte/innen sollten nach Einstieg in die Berufspraxis 
weiterhin an allgemeinen und medizinischen Sprachkursen teilnehmen. Besonders erfolgsversprechend scheint das Konzept des Sprachcoachings am jeweiligen Arbeitsplatz zu sein. ${ }^{56}$ Konzepte zur Integration ausländischer Ärzte/innen sollten eine entsprechende Entlastung und Flexibilität am Arbeitsplatz beinhalten.

Eine andere Herangehensweise ist das Angebot deutschsprachiger Medizinstudiengänge im Ausland. In Sachsen haben sich beispielsweise die Landesärztekammer, die Kassenärztliche Vereinigung, die Krankenhausgesellschaft und die AOK Plus im Wettbewerb um den ärztlichen Nachwuchs zusammengetan. Eine Maßnahme des Bündnisses ist die Finanzierung von zusätzlichen Studienplätzen (Verdopplung von 20 auf 40) für den deutschsprachigen Studiengang Humanmedizin an der Universität Pécs in Ungarn. ${ }^{57}$

Der Blick in die Berufspraxis einer Anästhesistin in Deutschland hat die hohe rechtliche und medizinische Relevanz differenzierter Deutschkenntnisse gezeigt. Eine Betrachtung weiterer Berufskontexte von Akademikern/innen würde sicherlich ebenfalls dazu beitragen, die Relevanz der Beherrschung der jeweiligen Nationalsprachen herauszustellen - speziell, wenn Experten-Laien-Kommunikation zum Berufsalltag gehört. Zumindest mit Blick auf die jeweils angestrebte Berufspraxis ist die Relevanz von Mehrsprachigkeit in der akademischen Bildung nicht zu unterschätzen.

\section{Literaturverzeichnis}

Aguado, Karin: Sozial-interaktionistische Ansätze. In: Deutsch als Fremd- und Zweitsprache. Ein internationales Handbuch. Bd. 1. Hgg. Ch. Fandrych, B. Hufeisen, H.-J. Krumm, C. Riemer. Berlin: De Gruyter 2010, S. 817-826.

Barkhuizen, Gary; Ellis, Rod: Analysing Learner Language. Oxford: Oxford University Press 2005.

Baur, Rupprecht; Chlosta, Christoph; Schäfer, Andrea: Fehleranalyse. In: Deutsch als Zweitsprache. 4. Aufl. Hgg. Bernt Ahrenholz, Ingelore Oomen-Welke. Baltmannsweiler: Schneider Verlag Hohengehren 2017, S. 353-368.

Borowski, Damaris: Dokumentation und kritische Reflexion des berufsbegleitenden Kurses >Deutsch für Ärzte/innen im Krankenhaus`. Hamburg: IQ-Landesnetzwerk 2014.

Borowski, Damaris: Sprachliche Herausforderungen ausländischer Anästhesisten/innen bei Aufklärungsgesprächen. Eine gesprächsanalytische Studie zu Deutsch als Zweitsprache im Beruf. Berlin: Frank \& Timme 2018. 
Bührig, Kerstin; Meyer, Bernd: Ärztliche Gespräche mit MigrantInnen. In: Handbuch Sprache in der Medizin. Hgg. Albert Busch, Thomas Spranz-Fogasy. Berlin u.a.: De Gruyter 2015, S. 300-316.

Bundesregierung: Entwurf eines Fachkräfteeinwanderungsgesetzes. Drucksache 19/8285 vom 13.3.2019. <http://dip21.bundestag.de/dip21/btd/19/082/1908285.pdf> (Zugriff: 26.6.2019).

Daase, Andrea; Ferber-Brull, Rosa; Kaplinska-Zajontz, Marta; Romero, Carlos Alejandro: Ein SPRUNQ-Brett auf dem Weg zum individuellen Ziel. Das Modellprojekt `Sprachcoaching für berufliche Unterstützung und Qualifizierung«. »Deutsch als Zweitsprache« 2 (2014), S. 6-23.

Ergebnisse der Ärztestatistik zum 31. Dezember 2018. Bundesärztekammer Berlin. $<$ https:// www.bundesaerztekammer.de/ueber-uns/aerztestatistik/aerztestatistik-2018> (Zugriff: 26.6.2019).

Gülich, Elisabeth; Mondada, Lorenza: Konversationsanalyse. Eine Einführung am Beispiel des Französischen. Tübingen: Max Niemeyer Verlag 2008.

Klüber, Maike: Verstehenssicherung zwischen Anästhesist und Patient im Aufklärungsgespräch. In: Handbuch Sprache in der Medizin. Hgg. Albert Busch, Thomas Spranz-Fogasy. Berlin u.a.: De Gruyter 2015, S. 208-224.

Klüber, Maike; Motsch, Johann; Spranz-Fogasy, Thomas: ıwenn sie sonst jetzt zum eingriff keine fragen mehr haben dann unterschreiben (.) sie noch mal hier،. Verständigungssicherung in anästhesiologischen Aufklärungsgesprächen - Arztangebote und Patientenfragen. »Deutsche Sprache« 40 (2012), S. 240-264.

Larsen, Reinhard (Hg.): Anästhesie. 10. Aufl. München: Urban \& Fischer in Elsevier 2013. Löning, Petra: Gespräche in der Medizin. In: Text- und Gesprächslinguistik. Ein internationales Handbuch zeitgenössischer Forschung. Bd. 1. Hgg. G. Antos, K. Brinker, W. Heinemann, S. Sager. Berlin: De Gruyter 2001, S. 1576-1588.

Mann, Frido: Aufklärung in der Medizin - Theorie, empirische Ergebnisse, praktische Anleitung. Stuttgart: F.K. Schattauer Verlag 1984.

Menz, Florian; Nezhiba, Sabine; Nowak, Peter; Rappl, Anita: Arzt-Patienten-Interaktion im deutschsprachigen Raum: Eine Online-Forschungsdatenbank (API-on®) als Basis für Metaanalysen. "Gesprächsforschung - Online-Zeitschrift zur verbalen Interaktion « 9 (2008), S. 129-163.

Neues Bündnis beschließt Maßnahmen gegen Ärztemangel in Sachsen. dpa/»Aerzteblatt.de«, 3.6.2019. <https://www.aerzteblatt.de/dossiers/aerztemangel?nid=103570> (Zugriff: 26.6.2019).

Nowak, Peter: Eine Systematik der Arzt-Patient-Interaktion. Systemtheoretische Grundlagen, qualitative Synthesemethodik und diskursanalytische Ergebnisse zum sprachlichen Handeln von Ärztinnen und Ärzten. Frankfurt/M. u.a.: Peter Lang Verlag 2010.

Schwitalla, Johannes: Gesprochenes Deutsch. Eine Einführung. 4. Aufl. Berlin: E. Schmidt 2012.

Selting, Margret et al.: Gesprächsanalytisches Transkriptionssystem 2 (GAT 2). »Gesprächsforschung - Online-Zeitschrift zur verbalen Interaktion« 10 (2009), S. 353-402.

Striebel, Walter H.: Anästhesie - Intensivmedizin - Notfallmedizin. Für Studium und Ausbildung. 9. Aufl. Stuttgart: Schattauer Verlag 2017. 